\title{
Variation Characteristics of Stem Water Content in Lagerstroemia indica and Its Response to Environmental Factors
}

\author{
Hao Liang $\mathbb{D}^{1,2,3}$ Meng Zhang, ${ }^{1,3}$ Hailan Wang $\mathbb{D}^{1,2,3}$ Chao Gao, ${ }^{4}$ and Yandong Zhao ${ }^{1,2,3}$ \\ ${ }^{1}$ School of Technology, Beijing Forestry University, Beijing 100083, China \\ ${ }^{2}$ Beijing Laboratory of Urban and Rural Ecological Environment, Beijing Municipal Education Commission, Beijing 100083, China \\ ${ }^{3}$ Research Center for Intelligent Forestry, Beijing 10083, China \\ ${ }^{4}$ School of Computer and Information Engineering, Beijing Technology and Business University, Beijing 100048, China
}

Correspondence should be addressed to Yandong Zhao; yandongzh@bjfu.edu.cn

Received 7 November 2019; Accepted 31 December 2019; Published 25 February 2020

Guest Editor: Zhenxing Zhang

Copyright (c) 2020 Hao Liang et al. This is an open access article distributed under the Creative Commons Attribution License, which permits unrestricted use, distribution, and reproduction in any medium, provided the original work is properly cited.

To achieve a rational allocation of limited water resources, and formulation of an appropriate irrigation system, this research studied the change characteristics of stem water content (StWC) in plant and its response to environmental factors. In this study, the StWC and environmental factors of Lagerstroemia indica in Beijing were continuously observed by a BD-IV plant stem water content sensor and a forest microclimate monitoring station from 2017 to 2018. The variation of StWC and its correlation with environmental factors were analyzed. The results showed that the StWC of Lagerstroemia indica varies regularly day and night during the growth cycle. Meanwhile, the rising time, valley time, and falling time of StWC were various at the different growth stages of Lagerstroemia indica. The results of correlation analysis between StWC and environmental factors indicated that the StWC of Lagerstroemia indica was positively correlated with air relative humidity, while it was negatively correlated with total radiation and air temperature. The multiple regression equation of StWC and environmental factors of Lagerstroemia indica was StWC $=11.789-1.402 \mathrm{Rn}-0.931 \mathrm{~T}-1.132 \mathrm{Ws}+0.933 \mathrm{RH}-3.368 \mathrm{ST}+2.168 \mathrm{SMC}$, and the coefficient of determination of the equation was of 0.87 . Furthermore, the results illustrated that the irrigation should pay attention to supplementing irrigation in time during the peak growing season of fruit.

\section{Introduction}

The stem is one of the two main structural axes of vascular plants, which plays a variety of roles in plants, such as mechanical support of leaves, flowers, and fruits, water transport between root and shoots in xylem and phloem, and water storage $[1,2]$. The variation of steam water content (StWC) is an important segment of water transport in soilplant-atmosphere continuum and also affects the transport of nutrients and the storage of carbohydrates. StWC is the result of interaction and feedback between different organs and their environment. A good understanding of StWC contributes to solving forestry research hotspots, such as cold resistance [3], drought resistance [4], and health assessment [5]. Moreover, accurately mastering the characteristics of
StWC and its environmental coupling mechanism is of great significance for formulating a reasonable irrigation system and making efficient use of water resources [6].

As a drought-resistant and pollution-resistant tree species, Lagerstroemia indica (Crape myrtle) has been widely used in the nursery cultivation and landscape use of cities in China. Water is one of the main factors restricting the growth and yield of Lagerstroemia indica; to assure the survival rate of Lagerstroemia indica, the irrigation strategies can be formulated more precisely according to the pattern of StWC of trees in different growth stages and the response to different environments, which also can improve the utilization rate of irrigation. In recent years, the research on the change of StWC in trees mainly focuses on fruit tree species such as jujube, walnut, and grape [7-9]. However, the research 
TABLe 1: Growth periods of Lagerstroemia indica.

\begin{tabular}{lccc}
\hline Flowering stage & Fruit growth stage & Fruit maturity stage & Dormancy stage \\
\hline $2017.3-2017.5$ & $2017.6-2017.8$ & $2017.9-2017.11$ & $2017.12-2018.2$ \\
\hline
\end{tabular}

on tree species used in landscape greening is rare. In addition, the relationship between StWC and environmental factors in the whole growth cycle of plants has not been thoroughly explored.

Researchers have proposed many methods for measuring the StWC of plants. Oven-drying method is a traditional way to estimate the water content in stem. The StWC can be directly calculated by measuring the weight difference between the original wet stem and the stem dried by an oven. The method is the most accurate but destructive way, which cannot be used for in situ detection and also cost a long period for measurement. Therefore, oven-drying is commonly used as a calibration of the other measurements. To achieve nondestructive and in situ measurement, some image analysis methods are introduced, including gamma-ray densitometry [10], X-ray computer tomography [11], and magnetic resonance imaging (MRI) [12]. Both gamma-ray densitometry and X-ray computer tomography are highly sensitive, which can acquire the StWC of plants accurately, while the potential safety risks restricted further application of them [13]. Although the MRI system can safely obtain the StWC of plants accurately, it still cannot achieve in situ and real-time detection, which is not suitable for long-term monitoring at a fixed site in the field. With the development of electronic detection technology, dielectric constant method has been widely used in StWC real-time and in situ monitoring, including time-domain reflection (TDR) technology [14-16] and frequency-domain (FD) [17-19] technology, and the TDR method has been the most commonly used. On the basis of TDR method, Constantz and Murphy used a parallel waveguide probe to detect the volume moisture content of pine stem [20]. Wullschleger and Hanson used a double-needle parallel stainless steel probe to monitor the seasonal variation of water content of four deciduous broad-leaved tree species. The calibration formulas suitable for the relationship between volume water content of stem and effective dielectric constant of tree species were summarized [21]. By summarizing the results of the previous studies of the researchers, it was feasible to detect StWC based on plant dielectric constant, but the length of the TDR probe restricted the detection effect. A short probe can reduce the damage to plants and the errors caused by the difference of water distribution, but the system resolution will be reduced, too. On the other hand, if the probes are too long, the signal attenuation will be very large. In addition, the TDR technology has high requirements and high production costs. Therefore, we used a nondestructive and real-time detection method of StWC on standing wave rate principle.

In this paper, Lagerstroemia indica growing in Beijing is selected as the research object. The area is affected by the cold wave, and the winter is cold and long, and the annual sunshine hours are significantly lower than other regions. The environmental conditions in Beijing vary widely, which leads the requirements of irrigation management for trees to be high. The aims of our research were to monitor the StWC of Lagerstroemia indica trees in situ continuously based on the theory of standing wave ratio (SWR) and analyze the variation characteristics of StWC and its response to meteorological factors at different scales. Furthermore, we studied the correlation between StWC and meteorological factors by using cross-correlation, path analysis methods, and modeling, to provide more empirical data for Lagerstroemia indica trees for planting and management by revealing the patterns of StWC changes. Our results will be of significance in the management of guiding the scientific irrigation of the nursery garden reasonably and efficiently and providing a scientific basis for the comprehensive evaluation of ecological and hydrological processes and influencing factors of garden tree species in Beijing, China.

\section{Materials and Methods}

2.1. Study Site. The study site is located in the Sanqingyuan nursery garden in Haidian District, Beijing, China $\left(40^{\circ} 0^{\prime} \mathrm{N}\right.$, $116^{\circ} 20^{\prime} \mathrm{E}$ ). The region is characterized by a semihumid warm temperate continental monsoon climate zone, with an annual temperature of $12.6^{\circ} \mathrm{C}$ and annual precipitation of $620 \mathrm{~mm}$, for which $80 \%$ of rainfall occurs primarily from June to August. With monthly percent possible sunshine ranging from $47 \%$ in July to $65 \%$ in January and February, the region receives 2,671 hours of bright sunshine annually. The mean annual evaporation of this area is $1,800 \mathrm{~mm}$, which is greater than precipitation by a factor of 3 . The climate information of the study site was collected by the forest microclimate monitoring station (School of Technology, Beijing Forestry University, Beijing, China). The height of the nursery is about 50 meters above sea level, and the terrain is flat, covering an area of about $31,200 \mathrm{~m}^{2}$. The soil of the site is clay loam with a pH between 7 and 8 . Vegetation at the site consists of deciduous broad-leaved trees dominated by Lagerstroemia indica, Juglans mandshurica, and Malus spectabilis.

2.2. Tree Material Preparation. We selected twelve Lagerstroemia indica tree samples with a similar diameter at breast height $(\mathrm{DBH})$ for stem water content monitoring, which were determined to be healthy, straight, nonstressed, and well-grown specimens, for each year of the experimental period. The DBH is a standard method of expressing the diameter of the trunk or bole of a standing tree, which is measured at $1.3 \mathrm{~m}$ above ground $[22,23]$. The DBH of the tree samples ranged from approximately $4.5 \pm 0.5 \mathrm{~cm}$, and the average tree height was $250 \pm 5 \mathrm{~cm}$. Irrigation in the site was carried out by small tube outflow, once a month for 1-2 days. In case of persistent high temperature and drought weather, irrigation was added 1-2 times a month. The amount of irrigation depended on the workers' experience. Weed control and pesticide spraying were carried out timely. The growth stages of Lagerstroemia indica trees are shown in 


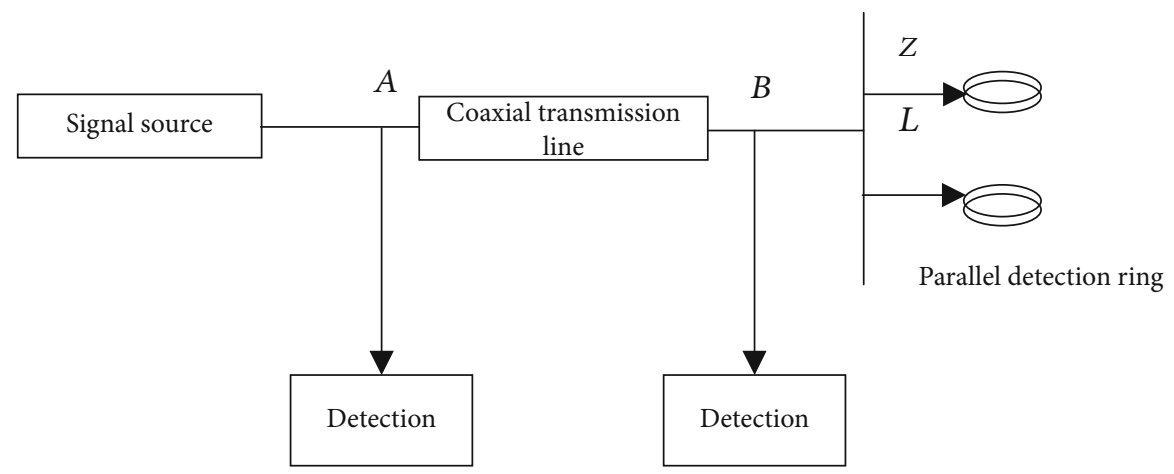

FIgURE 1: Schematic view of the BD-IV.

Table 1. Experiments were carried out under the existing conditions, and monitoring was performed from November 2017 to November 2018.

2.3. Stem Water Content Sensors. The BD-IV plant stem water content sensor (researched and developed by School of Technology, Beijing Forestry University, China) was implemented to measure the StWC of Lagerstroemia indica samples. The BD-IV, based on SWR, consisted of a $100 \mathrm{MHz}$ sinusoidal oscillator, a coaxial transmission line, a high-frequency detector circuit, and a pair of parallel stainless steel metallic electrodes [2, 24]. The electrodes were designed to wrap around stems like an upper and a lower strap ring (Figure 1). The electromagnetic wave provided by the sinusoidal oscillator spreads along the coaxial transmission line into the strap ring, and because the impedance of the strap ring is different from that of the transmission line, a proportion of the incident wave would be reflected back along the line to the oscillator. As a result of the reflected wave interfering with the incident wave, a voltage standing wave would be set up on the transmission line. According to the measuring principle of SWR, the differential voltage of $A$ and $B$ is taken as the output of the transducer conversion circuit, which can be expressed as a function of the strap ring impedance $[25,26]$ :

$$
U_{A B}=2 A_{1} \frac{Z_{L}-Z_{C}}{Z_{L}+Z_{C}}
$$

where $A_{1}$ is the amplitude of the electromagnetic wave (V), $Z_{L}$ is the impedance $(\Omega)$ of the strap ring, and $Z_{C}$ is the impedance $(\Omega)$ of the transmission line. In this study, the $Z_{C}$ is equal to $50 \Omega$. When $A_{1}$ and $Z_{C}$ are constant, the potential difference $U_{A B}$ at both ends of the transmission line is only related to the impedance of $Z_{L}$. The variation of StWC can affect the impedance of stem, which leads the change of the impedance of the strap ring $\left(Z_{L}\right)$, and the output voltage $\left(U_{A B}\right)$ of the sensor will be changed. In other words, $Z_{L}$ is determined by StWC of the measured living tree. Therefore, the StWC of living standing trees can be quantified by measuring the output voltage difference of the transmission line $\left(U_{A B}\right)$. (a)

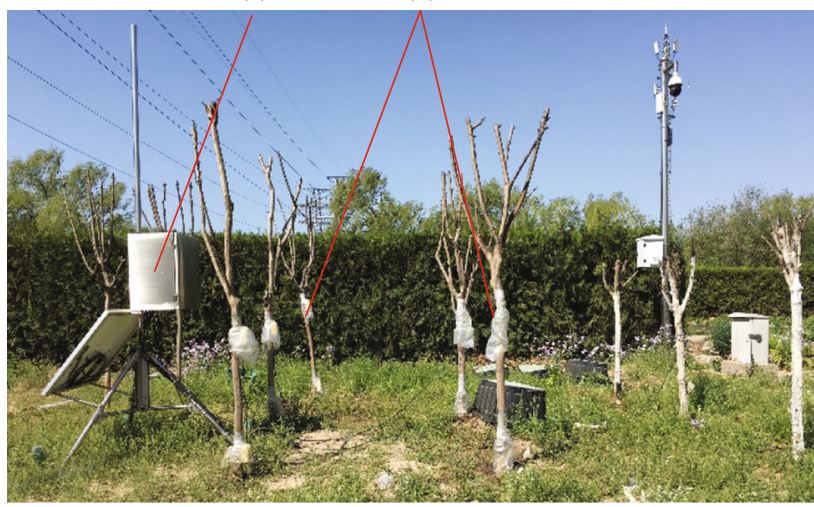

FIgURE 2: Monitoring station of stem water content: (a) digital collector and (b) SWR sensor (BD-IV).

2.4. Data Collection. StWC sensors (BD-IV) were installed on the trunks of 12 selected Lagerstroemia indica tree samples. The installation position of the BD-IV was close to the first bifurcation of the main trunk of the tree sample. A selfdesigned multichannel digital collector based on AVR 128 was used to connect the StWC sensors of each trunk. A monitoring station in the experiment is shown in Figure 2. The collector automatically collected and stored a data packet every 10 minutes and collected 144 data packets in one day. Thus, the collector can record 144 of StWC of each sample every day. During the whole experimental process, the same cultivation and management mode was adopted to ensure the supply of water and nutrients for all the samples. At the same time, the change process of StWC of the samples was recorded.

In the study site, a forest microclimate monitoring station (researched and developed by School of Technology, Beijing Forestry University, China) was built to measure the parameters of micrometeorological factors in the ecological microenvironment in real time, including air temperature, air relative humidity, global radiation, and wind speed. The station measured and collected the micrometeorological factors every 10 minutes. In addition, soil temperature sensors DS18B20 (Dallas Semiconductor, Dallas, Texas, United States) and soil volume moisture content sensors HYSWRARC (researched and developed by School of Technology, 


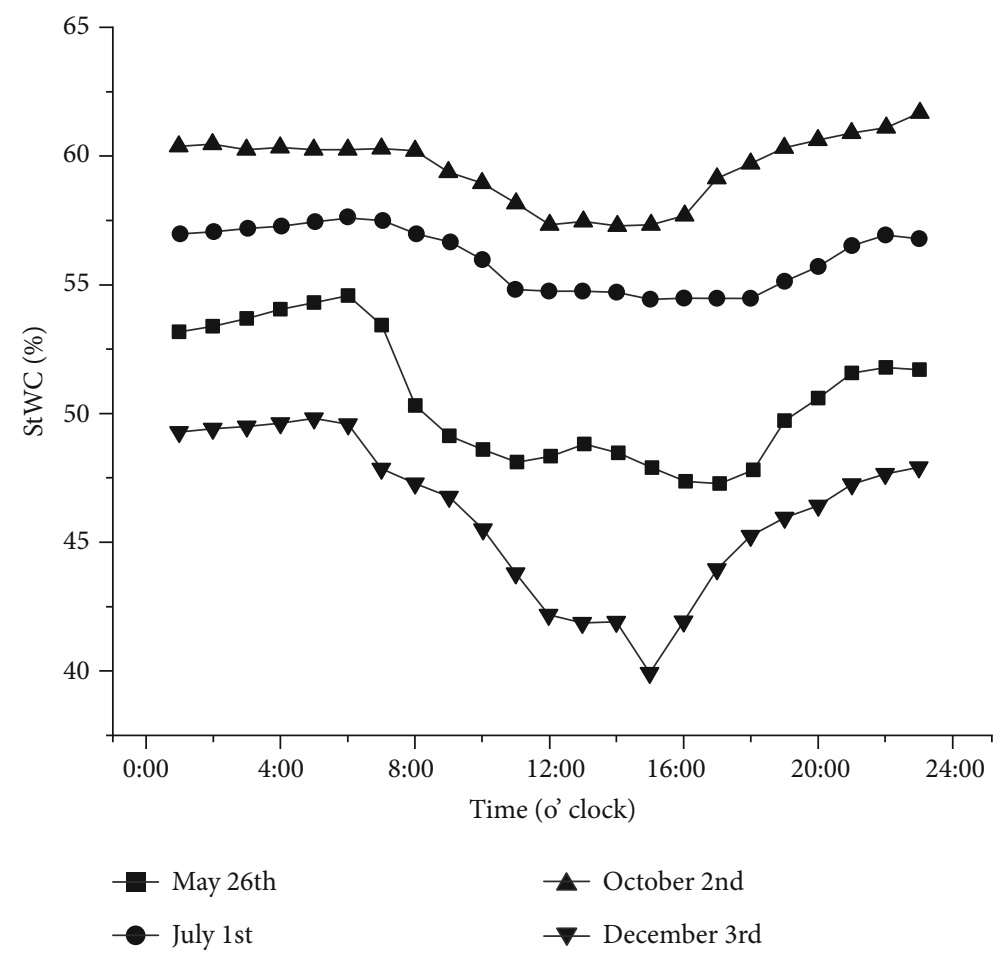

FIGURE 3: Daily variation of StWC in different growth stages of Lagerstroemia indica.

TABLE 2: Intermonthly dynamic change of StWC on sunny days and environmental factor daily mean of Lagerstroemia indica.

\begin{tabular}{|c|c|c|c|c|c|c|c|c|c|c|c|}
\hline \multirow{2}{*}{ Month } & \multirow{2}{*}{ Date } & \multicolumn{5}{|c|}{ Daily mean of environmental factor } & \multirow{2}{*}{ Falling time } & \multirow{2}{*}{ Valley time } & \multirow{2}{*}{ Valley value } & \multirow{2}{*}{ Rising time } & \multirow{2}{*}{ Mean StWC } \\
\hline & & $\mathrm{Rn}$ & $T$ & $\mathrm{RH}$ & Ws & $10 \mathrm{SMC}$ & & & & & \\
\hline 5 & 2018.5 .26 & 305.05 & 23.53 & 44.29 & 0.97 & 7.58 & 8:00 & $13: 00$ & 47.13 & $15: 30$ & 50.69 \\
\hline 7 & 2018.7.1 & 249.54 & 31.68 & 59.07 & 0.73 & 8.56 & 7:00 & $10: 30$ & 54.47 & 19:30 & 56.15 \\
\hline 10 & 2018.10 .2 & 185.59 & 16.01 & 60.19 & 0.61 & 9.98 & $6: 00$ & $10: 50$ & 57.23 & $18: 00$ & 59.78 \\
\hline 12 & 2018.12.2 & 139.78 & 0.95 & 50.85 & 0.50 & 7.66 & $7: 30$ & $12: 00$ & 39.79 & 14:00 & 46.18 \\
\hline
\end{tabular}

$\mathrm{Rn}$ : total radiation $\left(\mathrm{W} \cdot \mathrm{m}^{-2}\right) ; T$ : air temperature $\left({ }^{\circ} \mathrm{C}\right) ; \mathrm{RH}$ : relative humidity (\%); $10 \mathrm{SMC}: 10 \mathrm{~cm}$ soil moisture content (\%); falling time: time of StWC to fall; valley time: time of StWC to reach the minimum value; rising time: time of StWC to rise.

Beijing Forestry University, China) were installed at four different locations in the study site to monitor the soil temperature and soil volume moisture content, respectively.

2.5. Data Processing. The StWC of Lagerstroemia indica samples were calculated by

$$
V=1022.1 * \mathrm{St}+324.36 \quad\left(R^{2}=0.998\right)
$$

where $V$ is the output voltage difference of the transmission line and St is the StWC of Lagerstroemia indica samples. It is a linear relation between the StWC of the Lagerstroemia indica and the sensor output with the high sensitivity equal to $1022.1 \mathrm{mV}\left(\mathrm{cm}^{3} \cdot \mathrm{cm}^{-3}\right)^{-1}$. The value of coefficient of determination $\left(R^{2}\right)$ indicated that the sensor was able to measure the StWC of trees precisely.

Microsoft Excel (Microsoft Inc., Redmond, USA) was used to collate and calculate the StWC data of Lagerstroemia indica samples; SPSS 19.0 (SPSS Inc., Chicago, USA) and
MATLAB 2015b (MathWorks Inc., Natick, MA) were used to analyze the variance, regression, and correlation of the StWC and meteorological factors; and Origin (OriginLab Inc., USA) software was used to draw the charts and graphs.

\section{Results and Discussion}

3.1. Diurnal Variation Characteristics of Stem Water Content. The StWC data were selected from the typical sunny days. Figure 3 illustrates the diurnal variation characteristics of StWC of Lagerstroemia indica in its flowering stage (2017.5.26), fruit growth stage (2017.7.1), fruit maturity stage (2017.10.2), and dormancy period (2017.12.3). It can be found that the diurnal variation of StWC follows the overall trend of first decreasing and then increasing. Take the StWC data of October 2, 2017, as an example: StWC started to rapidly decrease at 6:00; after reaching the first trough at 11:00, the StWC was relatively stable till reaching the minimum value at 15:00; then, it began to increase smoothly. The rising 

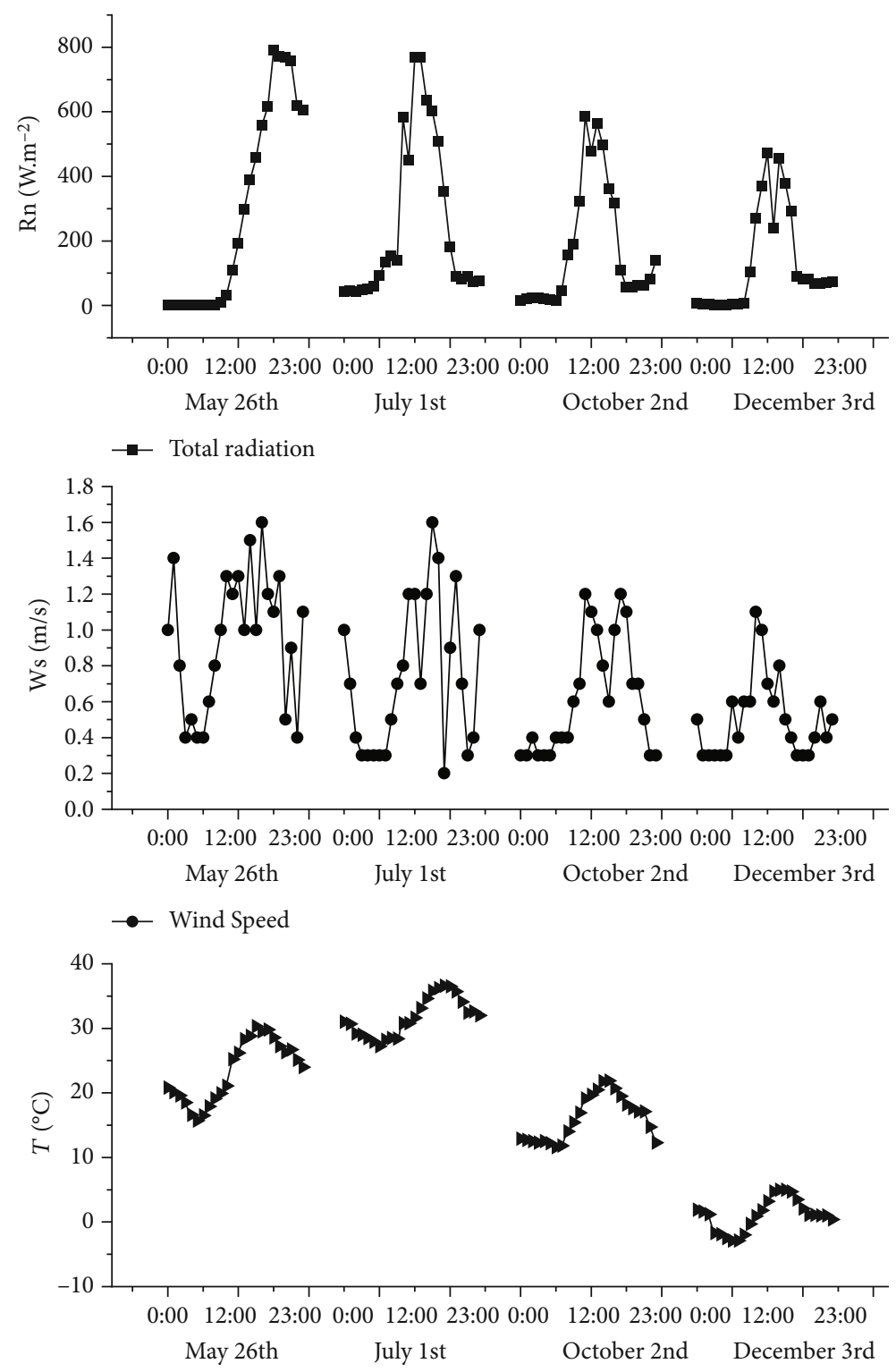

$\rightarrow$ Air temperature

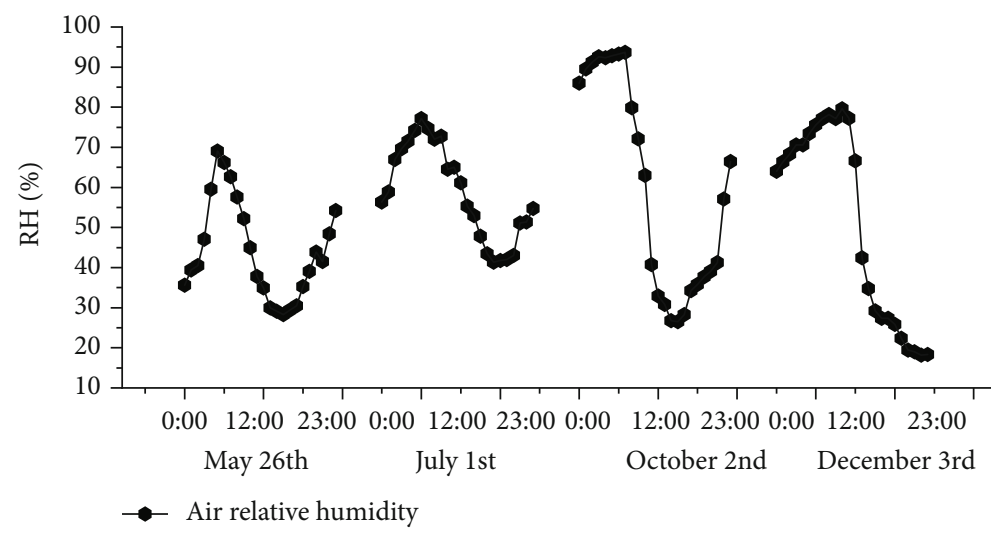

FIGURE 4: Daily variation of the main meteorological factors in different growth stages of Lagerstroemia indica.

process of StWC lasted nearly 16 hours until 5:00 the next day, and then, the falling process of StWC happened again. At different growth stages of Lagerstroemia indica, the valley time, falling time, and rising time of StWC were also variant. It can be seen from Table 2 that the valley time of StWC reached early, and the falling time of StWC started late in July 


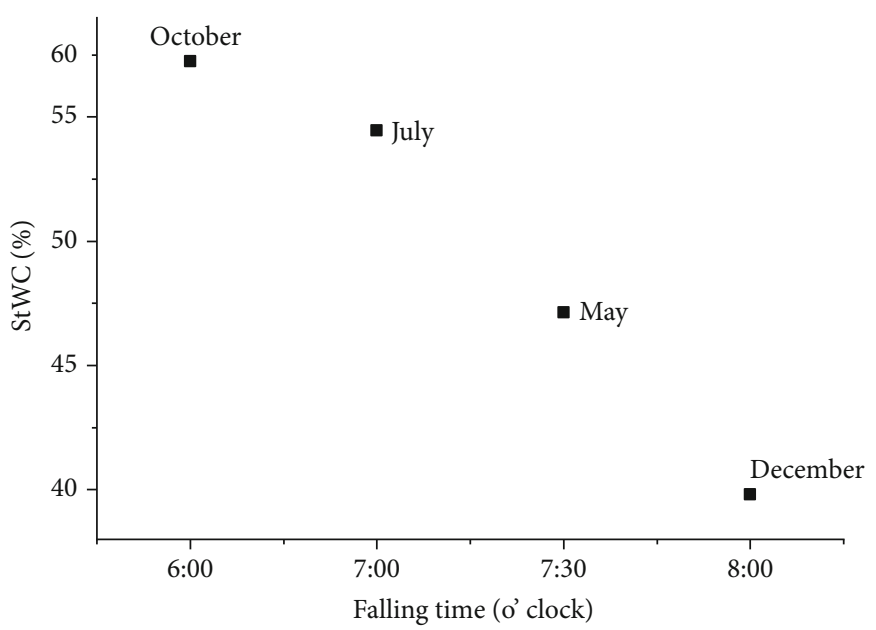

(a)

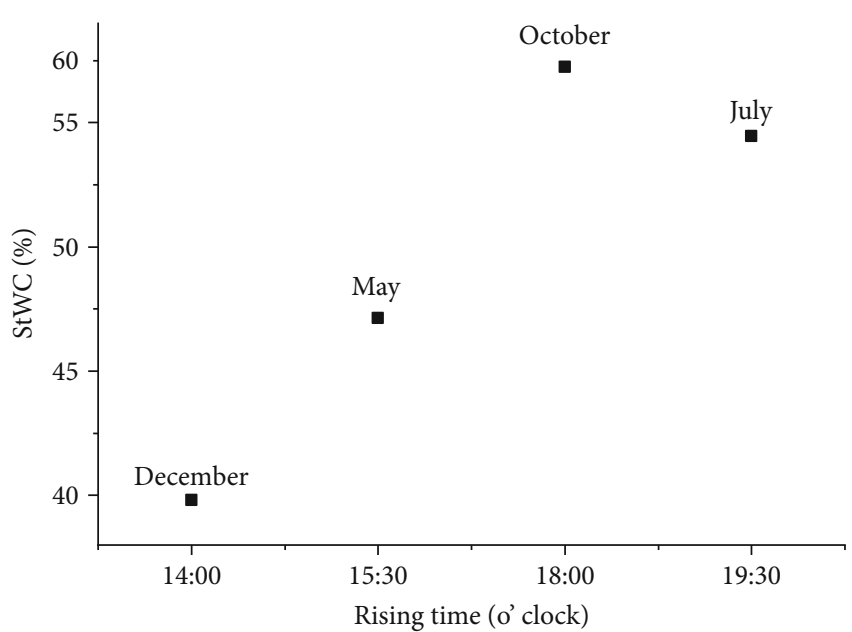

(b)

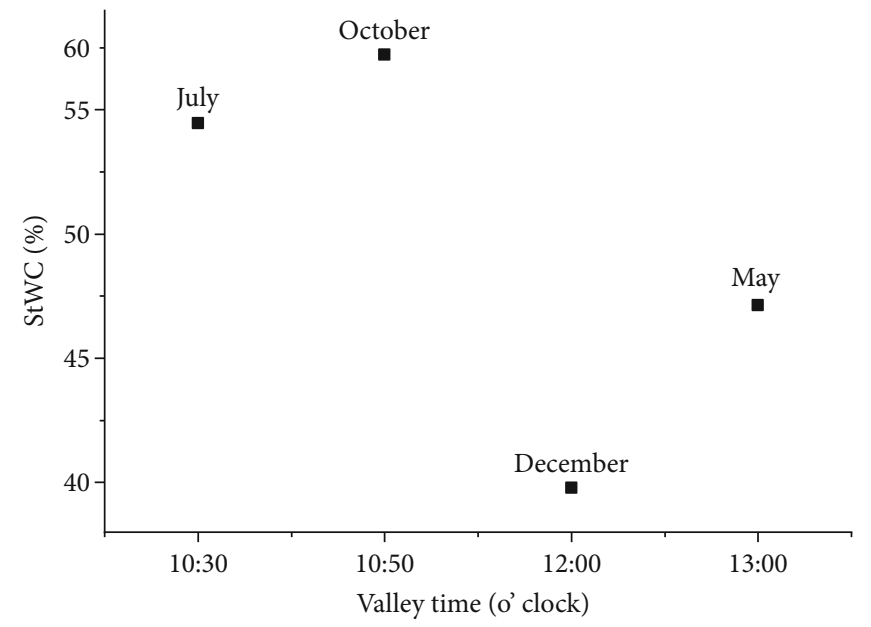

(c)

FIGURe 5: Relationship between valley StWC and StWC status. (a) Falling time of StWC. (b) Rising time of StWC. (c) Valley time of StWC.

and October. However, the pattern of StWC in March and December was conversely. This phenomenon may be related to the variation of meteorological factors during the growth period of the sample trees. The diurnal variation of the main meteorological factors on observation days at different growth periods is shown in Figure 4 . The results indicate that the falling time of StWC in the fruit growth stage and fruit maturity stage was early, and the rising time of StWC was late, which was conducive to the accumulation of photosynthates, while the opposite was found in the deciduous dormancy period and germination stage. Figure 5 shows the rising time, falling time, and valley time of StWC in the growth cycle of Lagerstroemia indica, which illustrates that the growth stage influenced the times of StWC greatly. The valley value of StWC was October $>$ July $>$ May $>$ December, the maximum StWC was $57.23 \%$ in October, and the minimum StWC was 39.79\% in December. In addition, the mean of StWC was October $>$ July $>$ May $>$ December, the maximum StWC was 59.78\% in October, and the minimum StWC was $46.18 \%$ in December. As a result, StWC of Lagerstroemia indica was the most at the fruit stage and the least at the deciduous dormancy period.
3.2. Response of Stem Water Content to Environmental Factors. The StWC of Lagerstroemia indica at different growth stages was significantly different under the influence of external environmental factors. We selected the data of StWC and meteorological factors for 7 consecutive days (from July 24 to 30, 2017) to analyze the relationship between StWC and environmental factors in Lagerstroemia indica. The variation curve of StWC and meteorological factors of Lagerstroemia indica is shown in Figure 6. It can be concluded that the StWC had strong correlation with total radiation, air temperature, air relative humidity, wind speed, and soil moisture content, but not with soil temperature. Moreover, StWC was contrary to that of total radiation, wind speed, and air temperature but similar to the change trend of relative air humidity, which indicated that the increase of air relative humidity would increase the StWC and have a negative effect on fruit tree transpiration, while the increase of total radiation and air temperature would decrease the water content of stem, which can promote the transpiration of Lagerstroemia indica. The photosynthate rate was influenced by the changes of radiation and air temperature in a reasonable range [27-29]. Since the tree samples were in 

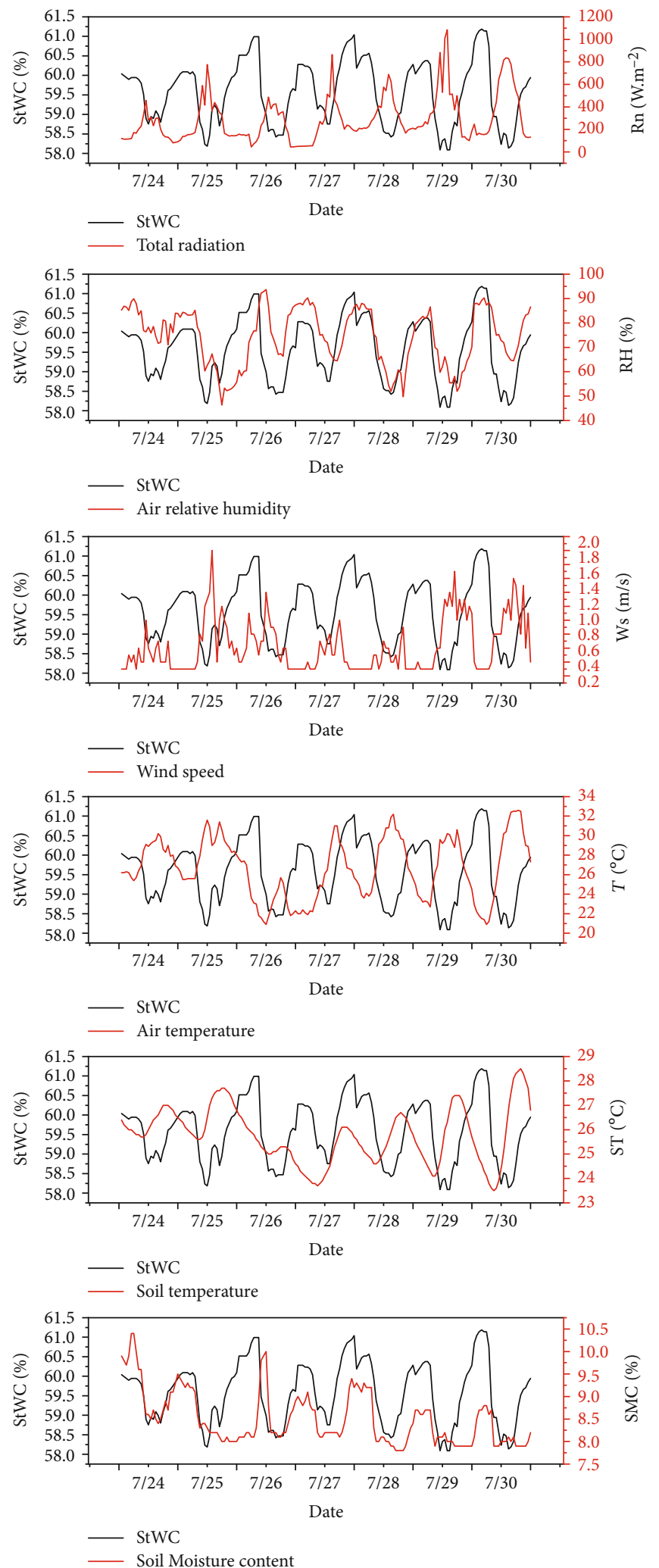

FIGURE 6: Relationship between StWC and environmental factors of Lagerstroemia indica.

the fruit growth stage, the accumulation of photosynthate would be increased with the increase of radiation and air temperature in a reasonable range [27-29].
The analysis results of correlation between StWC and environmental factors of Lagerstroemia indica are shown in Table 3. It can be seen that the StWC of Lagerstroemia 
TABLE 3: Correlation between StWC of Lagerstroemia indica and environmental factors.

\begin{tabular}{lcccccc}
\hline & Rn & RH & Ws & $T$ & ST & SMC \\
\hline Rn & 1 & & & & & \\
RH & $-0.455^{* *}$ & 1 & & & \\
Ws & $0.550^{* *}$ & $-0.530^{* *}$ & 1 & & \\
$T$ & $0.566^{* *}$ & $-0.718^{* *}$ & $0.481^{* *}$ & 1 & & \\
ST & -0.033 & $-0.475^{* *}$ & $0.405^{* *}$ & $0.681^{* *}$ & 1 & 1 \\
10 SMC & $-0.427^{* *}$ & $0.739^{* *}$ & $-0.488^{* *}$ & $-0.465^{* *}$ & $-0.194^{* *}$ & $-0.141^{* *}$ \\
StWC & $-0.732^{* *}$ & $0.505^{* *}$ & $-0.549^{* *}$ & $-0.556^{* *}$ & $0.414^{* *}$ & 1 \\
\hline
\end{tabular}

$\mathrm{Rn}$ : total radiation $\left(\mathrm{W} \cdot \mathrm{m}^{-2}\right)$; RH: air relative humidity $(\%) ; \mathrm{Ws}$ : wind speed $(\mathrm{m} / \mathrm{s}) ; T$ : air temperature $\left({ }^{\circ} \mathrm{C}\right) ; \mathrm{ST}$ : soil temperature $\left({ }^{\circ} \mathrm{C}\right) ; \mathrm{SMC}: 15 \mathrm{~cm}$ soil moisture content (\%); StWC: stem water content(\%). ${ }^{* *}$ Significant correlation at 0.01 level. ${ }^{*}$ Significant correlation at 0.05 level.

TABLE 4: Path analysis results between StWC and environmental factors.

\begin{tabular}{lccccccccc}
\hline Independent variable & $\begin{array}{c}\text { Simple correlation } \\
\text { coefficient }\end{array}$ & Direct path & \multicolumn{4}{c}{ Indirect path coefficient } \\
& coefficient & $X_{1}$ & $X_{2}$ & $X_{3}$ & $X_{4}$ & $X_{5}$ & $X_{6}$ & Total \\
\hline$X_{1}$ & -0.732 & -0.411 & - & 0.187 & 0.127 & 0.169 & 0.007 & 0.032 & 0.522 \\
$X_{2}$ & 0.505 & 0.137 & 0.187 & - & 0.122 & 0.219 & 0.106 & 0.055 & 0.689 \\
$X_{3}$ & -0.549 & -0.230 & 0.226 & 0.073 & - & 0.146 & 0.091 & 0.036 & 0.572 \\
$X_{4}$ & -0.556 & -0.305 & 0.229 & 0.098 & 0.111 & - & 0.153 & 0.034 & 0.625 \\
$X_{5}$ & -0.141 & 0.224 & 0.014 & 0.065 & 0.093 & 0.208 & - & 0.014 & 0.394 \\
$X_{6}$ & 0.414 & -0.074 & 0.175 & 0.101 & 0.112 & 0.142 & 0.043 & - & 0.573 \\
\hline
\end{tabular}

$X_{1}$ : total radiation $\left(\mathrm{W} \cdot \mathrm{m}^{-2}\right) ; X_{2}$ : air relative humidity $(\%) ; X_{3}$ : wind speed $(\mathrm{m} / \mathrm{s}) ; X_{4}:$ air temperature $\left({ }^{\circ} \mathrm{C}\right) ; X_{5}:$ soil temperature $\left({ }^{\circ} \mathrm{C}\right) ; X_{6}: 15 \mathrm{~cm}$ soil moisture content (\%).

indica was significantly correlated with total radiation, air temperature, air relative humidity, wind speed, soil temperature, and $15 \mathrm{~cm}$ soil moisture content at level 0.01 . The StWC was negatively correlated with air relative humidity and $15 \mathrm{~cm}$ soil moisture content but positively correlated with the other factors. The StWC had the strongest correlation with total radiation, and the correlation coefficient was 0.732. The degree of correlation was $r(\mathrm{Rn})=-0.732^{* *}$ $>r(T)=-0.556^{* *}>r(\mathrm{Ws})=-0.549^{* *}>r(\mathrm{RH})=0.505^{* *}$ $>r(10 \mathrm{SMC})=0.414^{* *}>r(\mathrm{ST})=-0.141^{* *} \quad\left({ }^{* *}\right.$ significant bilateral correlation at 0.01 level).

\subsection{Quantitative Correlation Analysis between Stem Water Content and Environmental Factors}

3.3.1. Multiple Regression Analysis. The experiment took the StWC of Lagerstroemia indica as dependent variable and environmental factors as independent variable and established a comprehensive relationship model between StWC and environmental factors based on the multiple regression analysis. The model was optimal to reflect the effect of environmental factors on StWC of Lagerstroemia indica. The multiple regression equation of StWC and environmental factors of Lagerstroemia indica is as follows:

$$
\begin{aligned}
\mathrm{StWC}= & 11.789-1.402 \mathrm{Rn}-0.931 T-1.132 \mathrm{Ws}+0.933 \mathrm{RH} \\
& -3.368 \mathrm{ST}+2.168 \mathrm{SMC},
\end{aligned}
$$

where St is the StWC of Lagerstroemia indica. The coefficient of determination of the equation is $0.87\left(R^{2}\right)$. The environmental factors could explain $87.0 \%$ of the StWC changes of Lagerstroemia indica. The coefficient of determination of the residual factor is $0.49\left(e=\sqrt{1-R^{2}}\right)$, which indicates that there are other factors having certain influence on StWC of Lagerstroemia indica, such as stomatal conductance and leaf area index.

Furthermore, the path analysis was implemented to study the way of influence and its magnitude of environmental factors on StWC of Lagerstroemia indica (Table 4). The direct effect of environmental factors on StWC is $\mathrm{Rn}(-0.411)>T$ $(-0.305)>\mathrm{Ws}(-0.230)>\mathrm{ST}(0.224)>\mathrm{RH}(0.137)>\mathrm{Hs}(-0.074)$, and the indirect effect of environmental factors on StWC is $\mathrm{RH}(0.689)>T$

$(0.625)>\mathrm{Hs}(0.573)>\mathrm{Ws}(0.572)>\mathrm{Rn}(0.522)>\mathrm{ST}(0.394)$. As a result, it shows that the direct effect of total radiation $(\mathrm{Rn})$ is the largest, while that of $15 \mathrm{~cm}$ soil moisture content (SMC) is the smallest. Air temperature mainly affected the StWC through the combined action of total radiation, relative humidity, and soil temperature. The total radiation mainly affected the StWC through the combined effects of air temperature, wind speed, and relative humidity.

\section{Conclusions}

This study confirms that the environmental factors can be significant predictors of daily stem water content (StWC) of 
Lagerstroemia indica in Beijing. According to the experimental results, the following conclusions can be drawn:

(1) The StWC of Lagerstroemia indica varies regularly day and night and follows the overall trend of first rising and then falling, which shows a typical valley curve. The rising time, valley time, and falling time of StWC are different at the different growth stages of the Lagerstroemia indica. Specifically, the falling time of the fruit growth stage and fruit maturity stage is ahead of the other stages, while the rising time of the fruit growth stage and fruit maturity stage is later than that of the other stages

(2) The StWC of Lagerstroemia indica is positively correlated with air relative humidity and $15 \mathrm{~cm}$ soil moisture content and negatively correlated with total radiation, wind speed, air temperature, and soil temperature which is the most important factor affecting StWC of Lagerstroemia indica. Moreover, the multiple regression equation of StWC and environmental factors of Lagerstroemia indica is $\mathrm{StWC}=11.789-1.402 \mathrm{Rn}-0.931 \mathrm{~T}-1.132 \mathrm{Ws}+$ $0.933 \mathrm{RH}-3.368 \mathrm{ST}+2.168 \mathrm{SMC}$, and the coefficient of determination of the equation is 0.87

(3) The transpiration of Lagerstroemia indica in the region of Beijing, China, is larger in June-September. The transpiration is highly influenced by environmental factors such as solar radiation and wind speed. The solar radiation is stronger in July and August in this region, and the air temperature is higher. Therefore, the irrigation should pay attention to supplementing irrigation in time during the peak growing season of fruit, i.e., during the period of fruit expansion, especially in the continuous sunny days of July and August. Irrigation frequency ensures that Lagerstroemia indica are not affected by water stress during the critical period of growth, and irrigation time should be chosen before sunrise or after sunrise to reduce the loss of irrigation water caused by evaporation

\section{Data Availability}

The data used to support the findings of this study are available from the corresponding author upon request.

\section{Conflicts of Interest}

The authors declare no conflict of interest.

\section{Authors' Contributions}

For this research article, Hao Liang and Yandong Zhao conceived and designed the experiments; Meng Zhang and Hailan Wang performed the experiments; Meng Zhang and Chao Gao analyzed the data; Hao Liang, Meng Zhang, and Hailan Wang wrote the paper; Hao Liang and Yandong Zhao reviewed and edited the paper.

\section{Acknowledgments}

This research was funded by the Fundamental Research Funds for the Central Universities (grant number BLX201717), the Special Fund for Beijing Common Construction Project, and the Science and Technology Program of the Inner Mongolia Autonomous Region (grant number 201802085).

\section{References}

[1] T. W. J. Scheenen, F. J. Vergeldt, A. M. Heemskerk, and H. van As, "Intact plant magnetic resonance imaging to study dynamics in long-distance sap flow and flow-conducting surface area," Plant Physiology, vol. 144, no. 2, pp. 1157-1165, 2007.

[2] C. Gao, Y. Zhao, and Y. Zhao, "A novel sensor for noninvasive detection of in situ stem water content based on standing wave ratio," Journal of Sensors, vol. 2019, Article ID 3594964, 10 pages, 2019.

[3] P. E. Verslues, M. Agarwal, S. Katiyar-Agarwal, J. Zhu, and J. K. Zhu, "Methods and concepts in quantifying resistance to drought, salt and freezing, abiotic stresses that affect plant water status," Plant Journal, vol. 45, no. 4, pp. 523-539, 2006.

[4] M. Farooq, A. Wahid, N. Kobayashi, D. Fujita, and S. M. A. Basra, "Plant drought stress: effects, mechanisms and management," Agronomy for Sustainable Development, vol. 29, no. 1, pp. 185-212, 2009.

[5] R. Spotts and A. Photosynthesis, "Photosynthesis, Transpiration, and water potential of apple leaves infected byVenturia inaequalis," Phytopathology, vol. 69, no. 7, pp. 717-719, 1979.

[6] A. Patakas, B. Noitsakis, and A. Chouzouri, "Optimization of irrigation water use in grapevines using the relationship between transpiration and plant water status," Agriculture Ecosystems \& Environment, vol. 106, no. 2-3, pp. 253-259, 2005.

[7] L. I. Hong, L. Bang, and L. I. Chang-Cheng, "Characteristics of stem flow of young jujube tree at different reproductive periods and their relationship with environmental factors," Agricultural Research in the Arid Areas, vol. 34, no. 5, pp. 54-61, 2016.

[8] Z. Fuyong, Z. Jinghua, and M. Yingjie, "The variation of stem flow and photosynthesis of walnut trees under drip irrigations," China Rural Water \& Hydropower, vol. 3, pp. 31-40, 2017.

[9] Y. Bai, G. F. Zhu, K. Zhang, and T. Ma, “Analysis of variation of sap flow velocity and water consumption of grapevine in the Nanhuoasis, Dunhuang," China. Journal of Desert Research, vol. 35, no. 1, pp. 175-181, 2015.

[10] W. R. N. Edwards and P. G. Jarvis, "A method for measuring radial differences in water content of intact tree stems by attenuation of gamma radiation," Plant Cell and Environment, vol. 6, no. 3, pp. 255-260, 1983.

[11] A. RASCHI, R. TOGNETTI, H.-. W. RIDDER, and C. BÉRES, "Water in the stems of sessile oak (Quercus petraea) assessed by computer tomography with concurrent measurements of sap velocity and ultrasound emission," Plant Cell \& Environment, vol. 18, no. 5, pp. 545-554, 2010.

[12] V. De Schepper, D. van Dusschoten, P. Copini, S. Jahnke, and K. Steppe, "MRI links stem water content to stem diameter variations in transpiring trees," Journal of Experimental Botany, vol. 63, no. 7, pp. 2645-2653, 2012. 
[13] H. G. Jones, "Irrigation scheduling: advantages and pitfalls of plant-based methods," Journal of Experimental Botany, vol. 55, no. 407, pp. 2427-2436, 2004.

[14] A. Nadler, E. Raveh, U. Yermiyahu, and S. R. Green, "Evaluation of TDR use to monitor water content in stem of lemon trees and soil and their response to water stress," Soil Science Society of America Journal, vol. 67, no. 2, pp. 437-448, 2003.

[15] M. Persson and S. Haridy, "Estimating water content from electrical conductivity measurements with short timedomain reflectometry probes," Soil Science Society of America Journal, vol. 67, no. 2, pp. 478-482, 2003.

[16] J. Irvine and J. Grace, "Non-destructive measurement of stem water content by time domain reflectometry using short probes," Journal of Experimental Botany, vol. 48, no. 3, pp. 813-818, 1997.

[17] N. M. Holbrook, M. J. Burns, and T. R. Sinclair, "Frequency and Time-Domain dielectric measurements of stem water content in the arborescent Palm,Sabal palmetto," Journal of Experimental Botany, vol. 43, no. 1, pp. 111-119, 1992.

[18] M. Stacheder, F. Koeniger, and R. Schuhmann, "New dielectric sensors and sensing techniques for soil and snow moisture measurements," Sensors, vol. 9, no. 4, pp. 2951-2967, 2009.

[19] W. Skierucha and A. Wilczek, "A FDR sensor for measuring complex soil dielectric permittivity in the $10-500 \mathrm{MHz}$ frequency range," Sensors, vol. 10, no. 4, pp. 3314-3329, 2010.

[20] J. Constantz and F. Murphy, "Monitoring moisture storage in trees using time domain reflectometry," Journal of Hydrology, vol. 119, no. 1-4, pp. 31-42, 1990.

[21] S. D. Wullschleger, P. J. Hanson, and D. E. Todd, "Measuring stem water content in four deciduous hardwoods with a timedomain reflectometer," Tree Physiology, vol. 16, no. 10, pp. 809-815, 1996.

[22] C. P. Brack, Standard point on tree bole for measurement. Forest measurement and modelling, Computer-based course resources for Forest Measurement and Modeling (FSTY2009) at the Australian National University, Canberra, Australia, 2009.

[23] T. R. Feldpausch, L. Banin, O. L. Phillips et al., "Heightdiameter allometry of tropical forest trees," Biogeosciences, vol. 8, no. 5, pp. 1081-1106, 2011.

[24] Y. Zhao, C. Gao, X. Zhang, and Q. Xu, "Non-destructive measurement of plant stem water content based on standing wave ratio," Transactions of the Chinese Society for Agricultural Machinery, vol. 40, pp. 310-316, 2016.

[25] G. J. Gaskin and J. D. Miller, "Measurement of soil water content using a simplified impedance measuring technique," Journal of Agricultural Engineering Research, vol. 63, no. 2, pp. 153-159, 1996.

[26] H. Zhou, Y. Sun, M. T. Tyree et al., "An improved sensor for precision detection of in situ stem water content using a frequency domain fringing capacitor," New Phytologist, vol. 206, no. 1, pp. 471-481, 2015.

[27] Z. Hong-jin, Z. Zhong-yuan, W. Xi-xi, S. Xiao-yuan, W. Hui, and J. Wei, "Effect of ecological factors and physiological factors on the net photosynthetic rate of Ulmus pumila at various growth stages," Acta Ecologica Sinica, vol. 36, pp. 1645-1651, 2016.
[28] Q. Han, T. Kawasaki, T. Nakano, and Y. Chiba, "Spatial and seasonal variability of temperature responses of biochemical photosynthesis parameters and leaf nitrogen content within a Pinus densiflora crown," Tree Physiology, vol. 24, no. 7, pp. 737-744, 2004.

[29] L. Gonzalez-Paleo and D. A. Ravetta, "Relationship between photosynthetic rate, water use and leaf structure in desert annual and perennial forbs differing in their growth," Photosynthetica, vol. 56, no. 4, pp. 1177-1187, 2018. 